\title{
Correction to: Refractory full thickness macular hole: current surgical management
}

\author{
Rino Frisina $(\mathbb{B}) \cdot$ Irene Gius $(\mathbb{D}) \cdot$ Luigi Tozzi $•$ Edoardo Midena $(\mathbb{B})$
}

Published online: 25 February 2022

(c) The Author(s), under exclusive licence to The Royal College of Ophthalmologists 2022

Correction to: Eye

https://doi.org/10.1038/s41433-020-01330-y
The original version of this article unfortunately contained a mistake. Figures 3 and 5 were incomplete. The corrected figures are given below. The original article has been corrected. 
Surgical technique

n. eyes FTMH closure rate n. $(\%)$

CI95\% Weight (\%)

Percentuage (\%)

Blood derivates subgroup (Autologous platelet concentrate $=$ APC, Plasma rich in growth factor $=$ PRGF)

1 Hillenkamp J et al ${ }^{18} \quad$ APC

2 Dimopoulos $\mathrm{S}$ et al ${ }^{46} \quad \mathrm{APC}$

3 Purtskhvanidze $\mathrm{K}$ et al ${ }^{47}$ APC

4 Arias JD et al ${ }^{48} \quad$ PRGC

17 (77)

$21(78)$

52 (85.2)

Lens capsular flap transplantation (LCFT) subgroup * whole blood

5 Chen $\mathrm{SN}$ et al ${ }^{62}$

LCFT

Peng J et al ${ }^{63}$ LCFT*

$2(100)$

54.6-92.2 5.2

57.7-91.4 6.4

73.8-93.0 14.1

15.8-100 0.7

0.7

$50.9-91.3 \quad 4.8$

$15(75)$

Autologous free internal limiting membrane flap (free ILM flap) subgroup * blood clot

7 Morizane $\mathrm{Y}$ et al ${ }^{49} \quad$ free ILM flap

8 De Novelli FJ et al ${ }^{50}$

free ILM flap
free ILM flap

pedicle ILM flap

9 Gekka T et al ${ }^{51}$

10 Park S W et al ${ }^{52}$

free ILM flap

11 Dai $Y$ et al ${ }^{53}$

12 Pires J et al ${ }^{54}$

free ILM flap

free ILM flap

free ILM flap

Ozdek Set

free ILM flap

15 Primavera $\mathrm{V}$ et al ${ }^{57}$

free ILM flap

6 Giansanti $\mathrm{F}$ et al ${ }^{58}$

17 Vieregge $\mathrm{M}$ et al ${ }^{59}$

free ILM flap

free ILM flap

18 Wu AL et al ${ }^{60}$

free ILM flap*

19 Soliman MK et al ${ }^{61}$

free ILM flap

$9(90)$

$55.5-99.7 \quad 2.5$

$4(80) \quad 28.4-99.5 \quad 1.4$

$3(100) \quad 29.2-100$

$2(100)$

2 (100)

12 (92.3)

11 (91)

$3(100)$

10 (90.9)

$5(100)$

$8(100)$

$3(100)$

$6(100)$

$4(80)$

15.8-100

15.8-100

64.0-99.8

61.5-99.8

29.2-100

$58.7-99.8$

47.8-100

63.1-100

29.2-100

54.1-100

28.4-99.5

\begin{tabular}{l|l}
5.2 \\
6.4 \\
4.1 \\
0.7
\end{tabular}

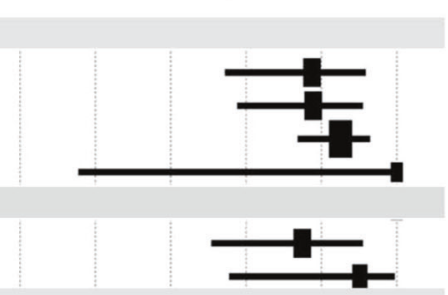

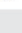

.4

0.9

0.7

0.7

3.2

3.0

0.9

2.7

1.4

2.1

2.1
0.9

1.6

5

Surgical technique

n. eyes FTMH closure rate n. (\%)

CI95\% Weight (\%)

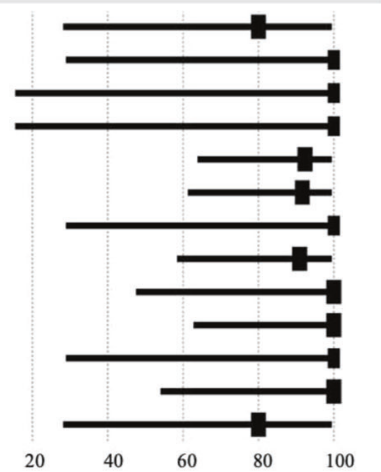

ID Authors

oup * in 4 eyes autologous serum

Enlargement of peeling subgroup * in 4 eyes autologous sce
20 D'Souza MJ et al ${ }^{19} \quad$ enlargement of peeling*

$21 \mathrm{Che} \mathrm{Xet} \mathrm{al}^{35} \quad$ enlargement of peeling

22 Hagiwara $\mathrm{A}$ et al ${ }^{36}$

enlargement of peeling

Macular hole hydrodissection (MHH) subgroup

23 Szigiato AA et al ${ }^{71} \quad$ MHH

24 Mohammed OA et al ${ }^{73}$ MHH

$25{\text { Felfeli } T \text { et al }{ }^{75} \quad \text { MHH }}^{75}$

26 Frisina $\mathrm{R}$ et al ${ }^{76}$

MHH

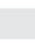

Autologous retinal graft (ARG) subgroup * blood clot ** whole blood in 5 eyes, viscoat in 5 (1)

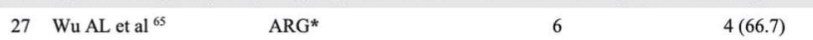
28 Chang $\mathrm{YC}_{\text {et al }}{ }^{66} \quad \mathrm{ARG}^{* *} \quad 10 \quad 9(90)$

Silicon oil (SO) subgroup (heavy $\mathrm{SO}=\mathrm{HSO}$, Light $\mathrm{SO}=\mathrm{LSO}$ ) * enlargement of ILM peeling

$\begin{array}{lll}29 & \text { Saeed MU et al }^{40} & \text { HSO* } \\ 31 & \text { Rizzo S et al }^{41} & \text { HSO } \\ 30 & \text { Lappas A et al }^{42} & \text { HSO } \\ 32 & \text { Cillino et al }^{43} & \text { HSO } \\ 33 & \text { Nowroozzadeh et al }^{39} & \text { LSO* }\end{array}$

33 Nowroozzadeh et

Human amniotic membrane (hAM) subgroup

34 Rizzo $\mathrm{S}$ et al ${ }^{67} \quad$ hAM

Perifoveal relaxing retinotomy subgroup

35 Reis $\mathrm{R}$ (t) ${ }^{68}$

Perifoveal relaxing retinotomy Arcuate temporal retinotomy subgroup

36 Charles $\mathrm{S}$ et al ${ }^{69} \quad$ Arcuate temporal retinotomy

Overall (fixed effects)
Overall (random effects)

$53(60)$

$23 \quad 20(86.9)$

$12 \quad 10(83.3)$

$119(81.8)$

$13 \quad 11(84.6)$

2 (48) $\quad 27.8-68.7$

(61.5) 31.6-86.1

$9(100)$

31.6-86.

(90) $\quad 55.5-99.7 \quad 2.5$

4 (100) $\quad 39.8-100 \quad 1.1$

$\begin{array}{lll}(83.3) & 51.6-97.9 & 3.0\end{array}$

$\quad 55.5-99.7$ 
ID Authors

Surgical technique

n. eyes BCVA gain logMAR mean (SE)

CI95\%

Weight (\%)

$\log M A R$

Blood derivates subgroup $($ Autologous platelet concentrate $=\mathbf{A P C}$, Plasma rich in growth factor $=\mathbf{P R G F}$ )

1 Hillenkamp $\mathrm{J}$ et al ${ }^{18} \quad$ APC

2 Dimopoulos $\mathrm{S}$ et al ${ }^{46} \quad$ APC

3 Purtskhvanidze $\mathrm{K}$ et al ${ }^{47}$ APC

4 Arias JD et al ${ }^{48}$ PRGC

$\begin{array}{cc}22 & -0.14(0.07) \\ 27 & -0.26(0.06) \\ 61 & -0.5(0.03) \\ 2 & -0.79(0.42)\end{array}$

$-0.27--0.01$
$-0.38--0.14$
$-0.55--0.45$
$-1.62-0.05$

Lens capsular flap transplantation (LCFT) subgroup * whole blood

5 Chen $\mathrm{SN}$ et al ${ }^{62}$

LCFT

6 Peng J et al ${ }^{63}$

LCFT*

$-0.46(0.09)$

$-0.5(0.15)$

$-0.63--0.29$

Autologous free internal limiting membrane (free ILM flap) subgroup * blood clot

7 Morizane $\mathrm{Y}$ et al ${ }^{49} \quad$ free ILM flap

8 De Novelli FJ et al ${ }^{50}$

Gekka $\mathrm{T}$ et al ${ }^{51}$

10 Park S W et al ${ }^{52}$

free ILM flap

11 Dai $Y$ et al ${ }^{53}$

pedicle ILM flap

free ILM flap

12 Pires J et al ${ }^{54}$

free ILM flap

free ILM flap

13 Guber J et al ${ }^{55}$

free ILM flap

4 Ozdek S et al ${ }^{56}$

15 Primavera $\mathrm{V}$ et al ${ }^{57}$

free ILM flap

free ILM flap

6 Giansanti $\mathrm{F}$ et al ${ }^{58}$

free ILM flap

Vieregge $\mathrm{M}$ et al ${ }^{59}$

free ILM flap

18 Wu AL et al ${ }^{60}$

free ILM flap*

$-0.43(0.1) \quad-0.63--0.23$

$-0.44(0.36)$

$-0.47(0.35)$

$-0.35(0.15)$

$-0.16(0.06)$

$-0.4(0.14)$

$-0.43(0.29)$

$-0.56(0.17)$

$-0.56(0.17)$

$-0.13(0.06)$

$-0.65(0.14)$

$-1.15-0.27$

$-1.16-0.22$

$-0.64--0.06$

$-0.27--0.05$

$-0.68--0.12$

$-1.01-0.15$

$-0.83--0.29$

$-0.88--0.24$

$-0.24--0.02$

$-0.93--0.37$

$-0.68--0.06$
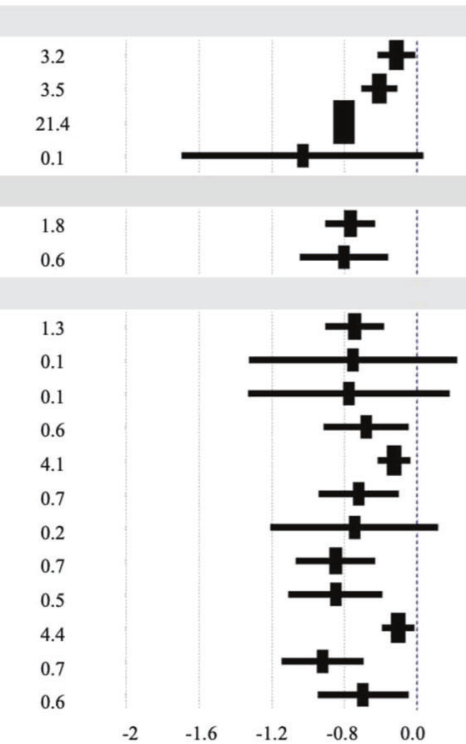

ID

Authors

Surgical technique

n. eyes BCVA gain logMAR mean (SE)

CI95\%

Weight (\%)

$\log M A R$

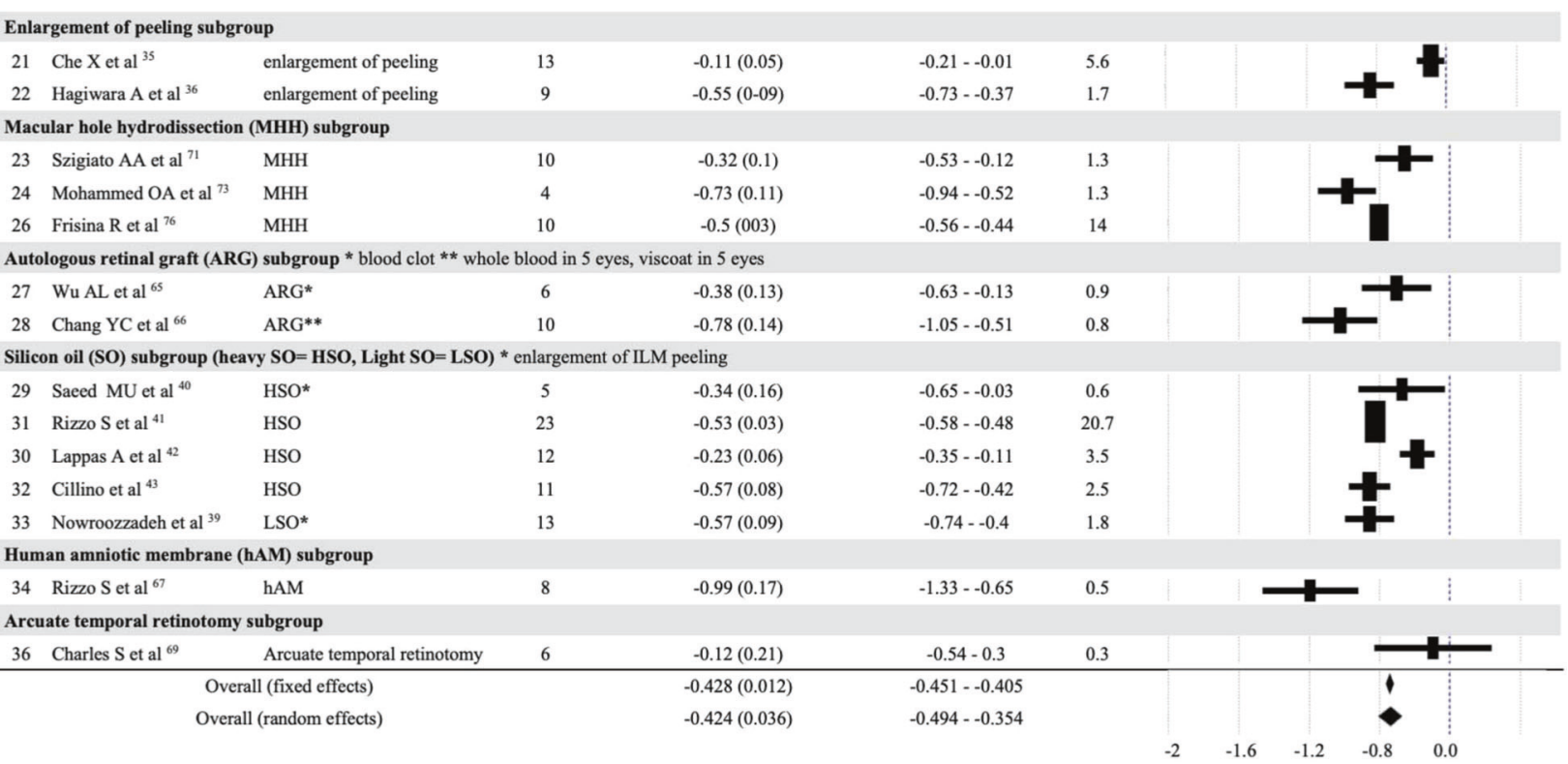

Heterogeneity test $Q=196.034, P<0.0001 ; I^{2}=84.19 \%(95 \% C I 78.62$ to 88.30$)$

Footnotes

$B C V A=B$ est corrected visual acuity. $\log M A R=$ logarithm of minimum angle of resolution. $S E=$ standard error. $C I=$ confidence interval

Fig. 5 Forest plot from meta-analysis of best-corrected visual acuity (BCVA) gain among the 32 analysed articles. Significant difference in BCVA gain among the surgical technique subgroups was demonstrated. 